\title{
Extragalactic neutrino background from very young pulsars surrounded by supernova envelopes
}

\author{
W. Bednarek \\ Department of Experimental Physics, University of Łódź, 90-236 Łódź, ul. Pomorska 149/153, Poland \\ Received 15 August 2001 / Accepted 10 September 2001

\begin{abstract}
We estimate the extragalactic muon neutrino background which is produced by hadrons injected by very young pulsars at an early phase after supernova explosion. It is assumed that hadrons are accelerated in the pulsar wind zone which is filled with thermal photons captured below the expanding supernova envelope. In collisions with those thermal photons hadrons produce pions which decay into muon neutrinos. At a later time, muon neutrinos are also produced by the hadrons in collisions with matter of the expanding envelope. We show that extragalactic neutrino background predicted by such a model should be detectable by the planned $1 \mathrm{~km}^{2}$ neutrino detector if a significant part of pulsars is born with periods shorter than $\sim 10 \mathrm{~ms}$. Since such population of pulsars is postulated by the recent models of production of extremely high energy cosmic rays, detection of neutrinos with predicted fluxes can be used as their observational test.
\end{abstract}

Key words. diffuse radiation: neutrinos - supernovae: general - pulsars: general - radiation mechanisms: non-thermal - cosmic rays

\section{Introduction}

The construction of high energy neutrino telescopes with the effective area $>10^{5} \mathrm{~m}^{2}$ (AMANDA $\rightarrow$ IceCube, ANTARES, NESTOR) will probably allow observation of first neutrinos of astrophysical origin, giving for the first time clear information about high energy hadronic processes in the universe. Therefore estimations of neutrino fluxes from discrete sources and their contribution to the extragalactic neutrino background (which may be probably easier to detect) is so important.

Up to now the flux of extragalactic neutrino background has been estimated with rather high confidence in the case of interaction of diffuse cosmic rays with the matter and radiation. Estimations of neutrino fluxes from discrete sources, where as it is well known, high energy processes are important i.e. active galactic nuclei and $\gamma$-ray bursts, are less certain. Also more exotic processes, like decays of topological defects, have been discussed as possible sources of extremely high energy neutrinos. For recent reviews of these estimations we refer to the articles by Protheroe (1998) and Learned \& Mannheim (2000).

Supernova explosions, during which young pulsars are formed, seem to be another likely sites of high energy processes in which observable neutrino fluxes are expected (e.g. Berezinsky \& Prilutsky 1978; Bednarek \& Protheroe 1997; Protheroe et al. 1998). In our recent paper

* e-mail: bednar@fizwe4.fic.uni.lodz.pl
(Beall \& Bednarek 2001, BB) we calculate the muon neutrino (and antineutrino) flux expected from a very young pulsar surrounded by the supernova envelope in our Galaxy. It has been shown that muon neutrinos are likely to be observed in the large scale $\left(1 \mathrm{~km}^{2}\right)$ neutrino detector from the supernova within our Galaxy if a pulsar with a period shorter than $\sim 10 \mathrm{~ms}$ is formed. However such explosions happen relatively rarely and we would have to be lucky to detect such a supernova within reasonable period of time. Therefore in order to test the predictions of the mentioned model by the planned neutrino detectors, we calculate in this paper the extragalactic neutrino flux produced by very young pulsars formed in the universe.

\section{Neutrinos from nuclei accelerated by pulsars}

Pulsars are produced during the type II and Ib/c supernova explosions of massive stars which detailed models have been investigated in many papers (see e.g. Woosley et al. 1993). When the iron core collapses, a very hot protoneutron star is formed. It cools to a neutron star within about $t_{\mathrm{NS}} \approx 5-10 \mathrm{~s}$ from the collapse (Burrows \& Lattimer 1986; Wheeler et al. 2000). The rest of the presupernova mass is expelled with the velocity of the inner radius of the order of $v_{1}=3 \times 10^{8} \mathrm{~cm} \mathrm{~s}^{-1}$ producing the supernova envelope. Its initial very high optical depth drops fast with time. The region between the pulsar and the envelope is filled with thermal radiation of characteristic temperature $T_{0} \approx 3 \times 10^{9} \mathrm{~K}$ (see Fig. 8 in 
Woosley et al. 1993). This radiation is not able to escape through the envelope because of its high optical depth. Assuming that the total energy of radiation inside the envelope is conserved, we can estimate the temperature of this radiation as a function of time during the expansion of the envelope $T(t)=T_{0}\left[R_{1} /\left(R_{1}+v_{1}\left(t_{\mathrm{NS}}+t\right)\right)\right]^{3 / 4}$, where $R_{1}=3 \times 10^{8} \mathrm{~cm}$ is the initial radius of the envelope at the moment of formation of the protoneutron star. When the pulsar forms (about $10 \mathrm{~s}$ after the explosion), the radiation temperature inside the envelope already drops to $\sim 5 \times 10^{8} \mathrm{~K}$. This radiation escapes through the envelope when the optical depth drops below $\sim 10^{3}$. This happens about $\sim 2 \times 10^{6}$ s after the explosion (see BB).

We assume that at the very early stage the pulsar loses energy only on electromagnetic waves. Therefore its period changes according to the formula $P_{\mathrm{ms}}^{2}(t)=$ $1.04 \times 10^{-9} t B_{12}^{2}+P_{0, \mathrm{~ms}}^{2}$, where $P_{0, \mathrm{~ms}} P_{\mathrm{ms}}$ are the initial and present periods of the pulsar, and $B_{12}$ is the pulsar surface magnetic field in units $10^{12} \mathrm{G}$. We neglect the pulsar energy losses to gravitational radiation due to the $r$-mode instabilities since they may become important about 1 year after pulsar formation (e.g. Andersson 1998; Lindblom et al. 1998).

It is argued that pulsars can accelerate particles to the highest energies observed in cosmic rays in the slingshot mechanism (see recent papers by e.g. Blasi et al. 2000 (BEO); De Goubeia Dal Pino \& Lazarian 2000; Bednarek $\&$ Protheroe 2001). Following work by Blasi et al. (BEO), we assume that a part of the magnetic energy in the pulsar's wind zone accelerates iron nuclei. The maximum energies, which nuclei can reach in the wind zone, depend on the pulsar parameters

$$
E_{\mathrm{Fe}}=\frac{B^{2}\left(r_{\mathrm{LC}}\right)}{8 \pi n_{\mathrm{GJ}}\left(r_{\mathrm{LC}}\right)} \approx 1.8 \times 10^{11} \beta B_{12} P_{\mathrm{ms}}^{-2} \mathrm{GeV},
$$

where $n_{\mathrm{GJ}}=B\left(r_{\mathrm{LC}}\right) /(2 e c P) \approx 3.3 \times 10^{11} B_{12} P_{\mathrm{ms}}^{-4}$ is Goldreich \& Julian (1969) density, $r_{\mathrm{LC}} \approx 4.77 \times$ $10^{6} P_{\mathrm{ms}} \mathrm{cm}, c$ is the velocity of light, and $\beta$ is the coefficient describing the efficiency of acceleration. According to the slingshot mechanism the acceleration of nuclei occurs very fast so that they do not lose energy during this stage. After acceleration, the nuclei move balistically through the radiation field (below the envelope) and later through the matter of the envelope. These particles are not frozen into the expanding plasma of the envelope and therefore do not lose energy on adiabatic process.

We apply the spectrum of iron nuclei accelerated in the pulsar wind zone close to the pulsar light cylinder derived in Beall \& Bednarek (BB). In this simple model the number of nuclei accelerated to energies $E$ scales as a part $\eta$ of the Goldreich \& Julian (1969) density at the light cylinder radius. It is assumed that the pulsar with specific parameters injects particles within some range of energies which is due to the fact that the magnetic field at different parts of the light cylinder radius is different. The energy spectrum of the iron nuclei below $E<E_{\mathrm{Fe}}$, which injected by the pulsar at a fixed age " $t$ " of the pulsar, is (see details in $\mathrm{BB}$ )

$$
\begin{aligned}
\frac{\mathrm{d} N}{\mathrm{~d} E \mathrm{~d} t}= & \frac{2 \pi c \eta r_{\mathrm{LC}}^{2} n_{\mathrm{GJ}}\left(E_{\mathrm{Fe}} E^{2}\right)^{-1 / 3}}{3\left[\left(E_{\mathrm{Fe}} / E\right)^{2 / 3}-1\right]^{1 / 2}} \\
& \cong \frac{3 \times 10^{30} \eta\left(B_{12} P_{\mathrm{ms}}^{-2} E^{-1}\right)^{2 / 3}}{\left[\left(E_{\mathrm{Fe}} / E\right)^{2 / 3}-1\right]^{1 / 2}} \frac{\mathrm{Fe}}{\mathrm{s} \mathrm{GeV}}
\end{aligned}
$$

Accelerated nuclei move in the pulsar wind almost at rest in the wind reference frame (BEO). Therefore we can neglect their synchrotron energy losses. However, as we mentioned above nuclei interact with strong thermal radiation field suffering complete photodesintegration before losing significant energies on $e^{ \pm}$pair and pion creation. For the considered parameters of the pulsar and the acceleration region, nucleons from desintegration of nuclei lose their energy mainly on pion production. Pions decay into neutrinos if their decay distance $\lambda_{\pi} \approx 780 \gamma_{\pi} \mathrm{cm}$, is shorter than their characteristic energy loss mean free path. The Lorentz factors of pions, $\gamma_{\pi}$, are comparable to the Lorentz factors of their parent nucleons, so they move similarly in the pulsar wind and their synchrotron losses do not dominate their inverse Compton scattering losses (ICS) in the thermal radiation. Pions lose energy on ICS process mainly in the Klein-Nishina (KN) regime, but not very far from the Thomson regime. By comparing the pion mean free path for ICS losses with their decay distance scale $\lambda_{\pi}$, we have found that pions can decay before losing energy on ICS if the temperature of thermal radiation drops below $T \sim 3 \times 10^{6} \mathrm{~K}$. The temperature of radiation below the envelope drops to this value about $t_{\mathrm{dec}} \sim 10^{4} \mathrm{~s}$ after supernova explosion. At this time nuclei are injected with the maximum energies allowed by Eq. (1) and cool in collisions with thermal radiation mainly on pion production. However when the optical depth through the expanding envelope drops below $\sim 10^{3}$ then the radiation is no more confined to the region below the envelope and its temperature drops much faster. Therefore we conclude that nucleons are able to cool efficiently in the radiation and produce pions which decay into muon neutrinos, only from $t_{\text {dec }} \approx 10^{4}$ s up to $t_{\text {conf }} \approx 2 \times 10^{6} \mathrm{~s}$ after explosion. At later times, the relativistic nuclei interact with the matter of the envelope which density is already low enough for the pions to decay into high energy neutrinos. Relativistic nucleons find enough column density of matter in the envelope $\left(>10 \mathrm{~g} \mathrm{~cm}^{-2}\right)$ to interact at the time shorter than about 1 year after explosion (see BB).

In terms of such a model we compute the spectrum of muon neutrinos from the pion decay, $N\left(E_{\nu}, P, B\right)$, produced by protons accelerated by pulsars with different periods, $P$, and surface magnetic fields, $B$ (see $\mathrm{BB}$ ). The pions are produced during the period $10^{4}-2 \times 10^{6} \mathrm{~s}$ after explosion as a result of complete cooling of nucleons in collisions with thermal radiation below the envelope, and during the period $2 \times 10^{6}-3 \times 10^{7} \mathrm{~s}$ from explosion as a result of collisions of iron nuclei with the matter of the envelope. 


\section{Extragalactic neutrino background}

In order to calculate the extragalactic neutrino background (ENB) we assume that the pulsars born at earier epoch do not differ significantly from those born at present. Thus the injection spectrum of neutrinos by a single pulsar depends only on its initial parameters (period, surface magnetic field) but not on redshift. Under this assumption, we can evaluate the neutrino injection rate at different redshifts by multiplying the injection spectrum of neutrinos of an average pulsar in our Galaxy by the neutron star formation rate, which depends on the massive star formation rate, which in turn depends on the redshift. In the previous section we have calculated the injection spectrum of neutrinos from the pulsar with known parameters (for details see BB). Let's determine now the neutrino injection rate from an "average" pulsar by integrating over the initial period and surface magnetic field distributions of pulsars within the Galaxy, according to

$Q\left(E_{\nu}\right)=\int_{P_{\min }}^{P_{\max }} \int_{B_{\min }}^{B_{\max }} N\left(E_{\nu}, P, B\right) f(P) \frac{\mathrm{d} N}{\mathrm{~d} B} \mathrm{~d} P \mathrm{~d} B$

where $P_{\min } \div P_{\max }$ is the range of initial periods of pulsars, $B_{\min }$ and $B_{\max }$ correspond to the range of typical values of the surface magnetic fields for the observed "classical" radio pulsars equal to $10^{11.5}$ and $10^{13.3}$, respectively.

It is believed that pulsars are born with rather short periods. However their exact period distribution is not known. Therefore we consider two possible distributions (see Giller \& Lipski 2001). The simplest case is described by the uniform distribution, $f(P)=1 /\left(P_{\max }-P_{\min }\right)$. And probably a more realistic case, described above $1 \mathrm{~ms}$ by the gamma function, which is defined by the mean pulsar period $\langle P\rangle$ and the index $\alpha, f(P)=A P^{\alpha} \mathrm{e}^{-(\alpha+1) P /\langle P\rangle}$, where $A=((\alpha+1) /\langle P\rangle)^{(\alpha+1)} / \alpha$ ! after normalization to one.

The distribution of surface magnetic fields of pulsars at birth can be estimated from the observed population of radio pulsars and assuming that the pulsar magnetic fields do not decay during the radio activity stage. It can be approximated by $\mathrm{d} N / \mathrm{d}(\log B) \approx 0.065 B_{12}^{-1}$ year $^{-1}$, for $B_{12}>2$, after normalization to the formation rate of pulsars in the Galaxy equal to 1 per 70 years (Narayan 1987). We estimate the distribution of pulsars with surface magnetic fields below $2 \times 10^{12} \mathrm{G}$ from Fig. 13 in Narayan (1987). This approximation may underestimate the number of pulsars with low magnetic fields, because of observational selection effects, and with high magnetic fields $>10^{13} \mathrm{G}$ since it does not take into account the so called "magnetars".

Now we can calculate the differential flux of ENB using the formula (see Fichtel \& Trombka 1981),

$N_{\nu}=\frac{c N_{\mathrm{G}}}{4 \pi H_{0}} \int_{0}^{z_{\max }} \frac{Q\left(E_{\nu}(1+z)\right) F(z)}{(1+z)\left(1+q_{0} z\right)^{1 / 2}} \mathrm{~d} z$,

where $z$ is the redshift, $N_{\mathrm{G}}=10^{-2} \mathrm{Mpc}^{-3}$ is the density of galaxies, $Q\left(E_{\nu}(1+z)\right)$ is the injection spectrum of neutrinos with energy $E_{\nu}(1+z)$, averaged over the distribution of initial pulsar periods and surface magnetic fields (see Eq. (3)). This is normalized to the pulsar birth rate per galaxy at the redshift $z=0$ equal to the one observed in our Galaxy. $f(z)$ is the evolution factor of the pulsar birth rate which we take proportional to the supernova rate (Kobayashi et al. 2000). Moreover we assume $H_{0}=75 \mathrm{~km} \mathrm{~s}^{-1} \mathrm{Mpc}^{-1}, q_{0}=0$, and $z_{\max }=5$.

The results of calculations of ENB are shown in Figs. 1 and 2, for the case of initial pulsar periods described by the uniform and gamma distributions, respectively. We investigate how the ENB depends on the parameters which define those distributions. In the case of uniform distribution of the initial periods we show the ENB from pulsars born within the range of periods limited by $P_{\min }=1 \mathrm{~ms}$ and $P_{\max }=100 \mathrm{~ms}, P_{\min }=1 \mathrm{~ms}$ and $P_{\max }=10 \mathrm{~ms}$, and $P_{\min }=10 \mathrm{~ms}$ and $P_{\max }=100 \mathrm{~ms}$. In the case of gamma distribution we show the ENB for $\langle p\rangle=10 \mathrm{~ms}$ and the in$\operatorname{dex} \alpha=2,10 \mathrm{~ms}$ and $\alpha=4$, and $50 \mathrm{~ms}$ and $\alpha=2$. In all cases $\eta=1$. Note that the ENBs, estimated for the pulsars which part has been born with periods below $10 \mathrm{~ms}$, are above the atmospheric neutrino background (ANB) produced in the interactions of cosmic ray particles with the earth's atmosphere. Therefore they can be potentially observed by future detectors.

\section{Discussion and conclusion}

We considered only neutrinos produced during the time when the supernova envelope is optically thick. However pulsars inject particles also after $>1$ year when the envelope becomes optically thin. Without detailed modeling of propagation of these particles in the supernova nebula it is difficult to conclude if the neutrino fluxes should be limited by the optically thick or thin neutrino bound marked in Figs. 1 and 2 (Waxman \& Bahcall 1999; Mannheim et al. 2001). The particles injected after 1 year can be captured by the magnetic field of the expanding large scale supernova nebula and suffer significant adiabatic energy losses (e.g. Bednarek \& Protheore 2001). The arguments that charged particles convert into neutrons, e.g. protons into neutrons, (see Waxman \& Bahcall 1999) may not be valid if the optical depth for hadronic collisions becomes low and adiabatic losses of captured hadrons are large.

At the end of the optically thick phase and during the optically thin phase, the pulsar-supernova envelopes should become also the sources of $\gamma$-rays and neutrons. Production of these neutral particles has been already considered in a similar supernova model (with the difference that particles are accelerated in the inner pulsar magnetosphere, Protheroe et al. 1998; Bednarek \& Protheroe 1997). The contribution of these neutral particles to the $\gamma$-ray background and cosmic rays in terms of the present model will be discussed in a future paper.

We have discussed also the problem whether the estimated extragalactic neutrino background from the whole population of pulsars in the universe can be detected by the planned experiments. The full horizontal line in Figs. 1 


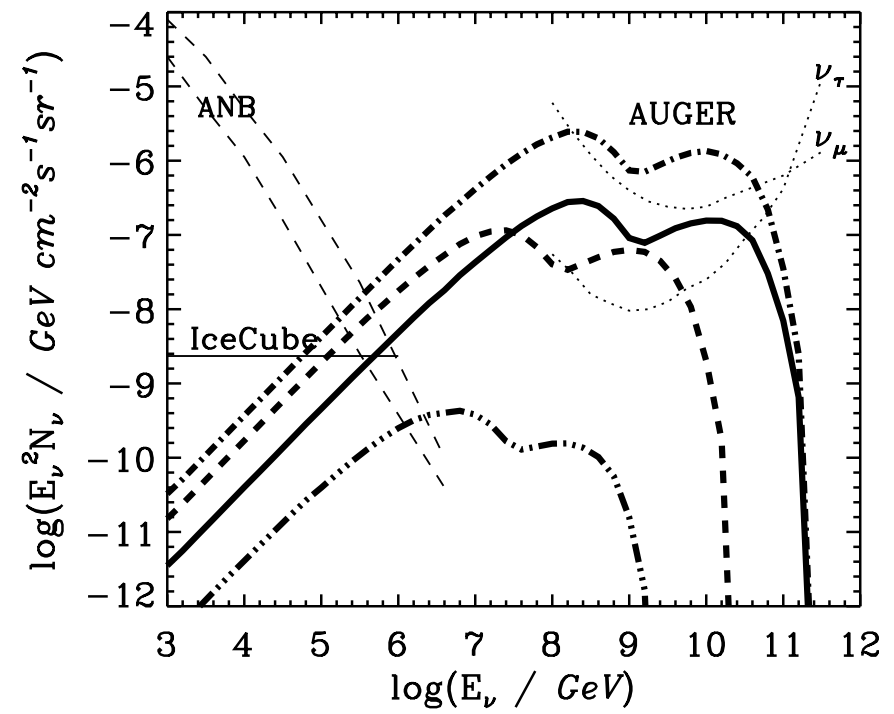

Fig. 1. The extragalactic neutrino background produced by pulsars with uniform distribution of initial periods between 1-10 ms (dot-dashed curve), 1-100 ms (full curve), 10-100 ms (dot-dot-dot-dashed curve) and the acceleration parameter $\beta=1$, and $1-10 \mathrm{~ms}$ and $\beta=0.1$ (thick dashed curve). The upper bounds on extragalactic neutrinos for the case of optically thick (MP\&R, Mannheim et al. 2001) and thin sources (W\&B, Waxman \& Bahcall 1999) are marked by the horizontal thin dot-dashed lines. The atmospheric neutrino background (ANB) is shown by the dashed curves. The sensitivity of the planned IceCube detector is marked by the horizontal thin full line (Spiering 2000), and the AUGER array by the thin dotted curves for the muon $\nu_{\mu}$ and tau $\nu_{\tau}$ neutrinos (Bertou et al. 2001).

and 2 shows the detection limit of the IceCube detector (Spiering 2000). It looks that ENB from pulsars should be easily observed by the IceCube if significant part of pulsars are born with initial periods shorter than $\sim 10 \mathrm{~ms}$ in the case of uniform distribution (see Fig. 1), and if the mean pulsar period is close to $10 \mathrm{~ms}$ in the case of the gamma distribution (see Fig. 2), assuming that $\eta \sim 1$. Also the planned AUGER experiment with the detection limits estimated by Bertou et al. (2001) may observe ENB from pulsars if the significant amount of pulsars is born with the initial periods of the order of a few $\mathrm{ms}$ as postulated by the recent models for the pulsar origin of the highest energy cosmic rays (BEO, De Goubeia Dal Pino \& Lazarian 2000). However, such detection would be possible if the hypothesis of $\nu_{\mu} \rightarrow \nu_{\tau}$ oscillations with full mixing is correct. In such case the expected flux of tau neutrinos, compared to the calculated flux of muon neutrinos, is above the sensitivity limit of the AUGER detector for the case of pulsars born with the milisecond periods (see lower dotted curve in Fig. 1, taken from Bertou et al. 2001).

Acknowledgements. I thank the referee for comments and suggestions. This work is supported by the Polish KBN grant No. 5P03D02521.

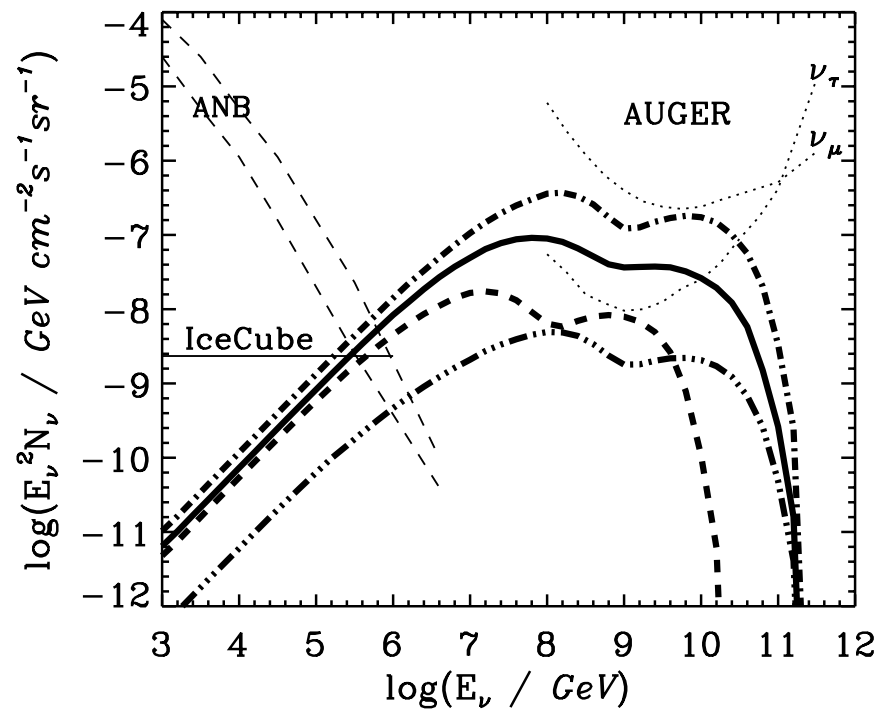

Fig. 2. As in Fig. 1 but for pulsars with the initial periods described above $1 \mathrm{~ms}$ by the gamma function with the mean period $\langle p\rangle=10 \mathrm{~ms}$ and index $\alpha=2$ (dot-dashed curves), $10 \mathrm{~ms}$ and $\alpha=4$ (full curves), and $50 \mathrm{~ms}$ and $\alpha=2$ (dot-dot-dotdashed curves) ( $\beta=1$ in the above cases), and for $\langle p\rangle=10 \mathrm{~ms}$, $\alpha=2$, and $\beta=0.1$ (thick dashed curve).

\section{References}

Andersson, N. 1998, ApJ, 502, 708

Beall, J. H., \& Bednarek, W. 2001, ApJ, submitted (BB)

Bednarek, W., \& Protheroe, R. J. 1997, PRL, 79, 2616

Bednarek, W., \& Protheroe, R. J. 2001, Astropart. Phys., in press

Berezinsky, V. S., \& Prilutsky, O. F. 1978, A\&A, 66, 325

Bertou, X., Billoir, P., Deligny, O., Lachaud, C., \& LetessierSelvon, A. 2001, Astropart. Phys., in press

Blasi, P., Epstein, R. I., \& Olinto, A. V. 2000, ApJ, 533, 123 (BEO)

Burrows, A., \& Lattimer, J. M. 1986, ApJ, 307, 178

De Goubeia Dal Pino, E. M., \& Lazarian, A. 2000, ApJ, 536, L31

Fichtel, C. E., \& Trombka, J. I. 1981, Gamma Ray Astrophysics (NASA SP-453, Washington, DC), 192

Giller, M., \& Lipski, M. 2001, Proc. 28th ICRC (Hamburg), 2092

Kobayashi, C., Tsujimoto, T., \& Nomoto, K. 2000, ApJ, 539, 26

Learned, J. G., \& Mannheim, K. 2000, Ann. Rev. Nucl. Part. Sci., 50, 679

Lindblom, L., Owen, B. J., \& Morsink, S. M. 1998, PRL, 80, 4843

Mannheim, K., Protheroe, R. J., \& Rachen, J. P. 2001, Phys. Rev. D, 63, 23003

Narayan, R. 1987, ApJ, 319, 162

Protheroe, R. J. 1998 [astro-ph/9809144]

Protheroe, R. J., Bednarek, W., \& Luo, Q. 1998, Astropart. Phys., 9, 1

Spiering, C. 2000, Nucl. Phys. Suppl., 91, 445

Waxman, E., \& Bahcall, J. 1999, Phys. Rev. D, 59, 023002

Wheeler, J. C., Yi, I., Höflich, P., \& Wang L. 2000, ApJ, 537, 810

Woolsey, S. E., Langer, N., \& Weaver, T. A. 1993, ApJ, 411, 823 